\title{
Drug output of unvented jet nebulizers as a function of time
}

\author{
H. Diederik ${ }^{\mathrm{a}, 1}$, P.P.H. Le Brun ${ }^{\mathrm{b}}$, H.W. Frijlink ${ }^{\mathrm{c}}$, P.M.B. Vitányi ${ }^{\mathrm{d}}$, \\ M. Weda ${ }^{\mathrm{a}, 1}$, D.M. Barends ${ }^{\mathrm{a}, *, 1}$ \\ ${ }^{a}$ RIVM, National Institute of Public Health and the Environment, Antonie van Leeuwenhoeklaan 9, \\ P.O. Box 1, 3720 BA Bilthoven, The Netherlands \\ b The Hague Hospitals Central Pharmacy, The Hague, The Netherlands \\ ${ }^{\mathrm{c}}$ Department of Pharmaceutical Technology and Biopharmacy, University of Groningen, Groningen, The Netherlands \\ ${ }^{\mathrm{d}}$ National Center of Mathematics and Computer Science (CWI), Amsterdam, The Netherlands
}

Received 10 December 2001; received in revised form 22 January 2003; accepted 29 January 2003

\begin{abstract}
Nebulizer drug output rate increases during the nebulization. For unvented jet nebulizers, a physical and mathematical model based on the efficiency of the nebulization process is presented for this phenomenon. Formulas are derived for the cumulative drug output and the drug output rate of the nebulization process. The model is compared with the model proposed by Coates et al. [J. Aerosol. Med. 11 (1998) 101]. Both models are supported by experimental literature data. Both models predict the experimental values well but the proposed model allows more easy prediction of the influence of small changes in the nebulization conditions and the calculation of the cumulative drug output for a related process. From literature data it is shown that the efficiency of an unvented jet nebulization process of diluted aqueous solutions is relatively insensitive to small changes in the concentration as well as to small changes in aspiration flow but is sensitive to the humidity of the compressor gas only.
\end{abstract}

(C) 2003 Elsevier Science B.V. All rights reserved.

Keywords: Jet nebulizer; Drug output; Efficiency

\section{Introduction}

When an aqueous solution is nebulized an increase in concentration of the solution is observed. This is caused by evaporation of the solvent in addition to the nebulization of the drug solution and is known as evaporative loss (Coates et al., 1998a). For instance, with the Cirrus, an unvented jet nebulizer, an increase from $10.0 \%, \mathrm{~m} / \mathrm{v}$ up to $13.1 \%, \mathrm{~m} / \mathrm{v}$ after $12 \mathrm{~min}$ was

\footnotetext{
* Corresponding author. Tel.: +31-30-274-4209; fax: +31-30-274-4462.

E-mail address: dirk.barends@rivm.nl (D.M. Barends).

1 Assessor for the Medicines Evaluation Board in The Netherlands. This paper does not necessarily reflect the opinion of the Medicines Evaluation Board in The Netherlands.
}

reported (Le Brun et al., 1999a). Similar experimental observations were reported by other authors (Coates et al., 1998a; Raabe et al., 1998). Due to the increase in drug output rate over time and by the existence of a residual volume that cannot be nebulized it is not easy to determine the nebulization time needed to deliver a prescribed dose and the necessary dilution-if any-of the drug solution.

A simple way-out to correct for the increase of the drug output rate over time is the use of the mean drug output rate and the mean cumulative drug output. A more accurate method is to describe this phenomenon by a mathematical formula.

For unvented nebulizers, such a mathematical relationship was established by superimposing the expo- 
nential process of the increase of concentration onto the linear process of the loss of volume of nebulization solution (Coates et al., 1998a). These authors presented formulas for the cumulative drug output, drug output rate and volume; these formulas were experimentally validated. However, the parameters in their formulas are not easily interpreted. Consequently, it is not clear if parameters measured for some process can also be used to describe a related nebulization process.

Other authors described the change in concentration in an unvented jet nebulizer by a formula in which the so-called efficiency of the process is used (Raabe et al., 1998). The efficiency is the ratio of the rate of nebulization of the drug solution to the rate of total volume loss. These authors also demonstrated the validity of their formula experimentally but did not give a derivation. Also, no formulas were given for cumulative drug output and drug output rate based on this physical model.

In this paper, we present a derivation of the formula of Raabe and, based on this efficiency-model, we present formulas for:

- the concentration in the nebulization solution at a given nebulization time ((1), see below),

- the cumulative nebulized dose after a given nebulization time ((2), see below),

- the nebulization time required to generate a prescribed dose ((3), see below),

- the remaining volume in the nebulizer at a given nebulization time ((4), see below).

\section{Output time dependency}

We define:

$t$ time

$V_{0} \quad$ volume of the solution in the nebulizer at the start of the nebulization

$C_{0} \quad$ drug concentration in the solution at the start of the nebulization

$V_{t} \quad$ volume of the solution in the nebulizer at time $t$

$C_{t}$ drug concentration in the solution at time $t$

$\alpha \quad$ volume of drug solution nebulized per unit of time

$\psi \quad$ volume of drug-free solvent evaporated per unit of time

$D_{t} \quad$ cumulative drug output at time $t$.
The total volume output per unit of time of the nebulization process is $(\alpha+\psi)$; the efficiency of the nebulizer is $\alpha /(\alpha+\psi)$. The efficiency depends on the type of nebulizer, on the composition of the nebulization solution, on the aspiration of the nebulized aerosol, the temperature and for jet nebulizers also on the relative humidity of the jet gas (Coates et al., 1998a; Raabe et al., 1998). This is discussed into more detail below. As a first approximation, we assume that $\alpha$ and $\psi$ are constant during nebulization. This is an approximation only, as during nebulization the concentration increases, hence the viscosity may increase. Also, the temperature decreases in jet nebulizers. These factors all affect the efficiency. However, the approximation that $\alpha$ and $\psi$ are constant seems to be acceptable for our goals by the fair correspondence of the results computed from our formulae and the experimental data, see below.

In Appendix A the following formulas are derived:

$C_{t}=C_{0}\left[\frac{V_{0}}{V_{0}-(\alpha+\psi) t}\right]^{\psi /(\alpha+\psi)}$

$D_{t}=C_{0} V_{0}\left\{1-\left[\frac{V_{0}-(\alpha+\psi) t}{V_{0}}\right]^{\alpha /(\alpha+\psi)}\right\}$

$t_{D}=\frac{V_{0}}{\alpha+\psi}\left\{1-\left[1-\frac{D}{C_{0} V_{0}}\right]^{1+\psi / \alpha}\right\}$

$V_{t}=V_{0}-(\alpha+\psi) t$

Formula (4) enables to calculate the residual volume once the total nebulization time needed to deliver a prescribed dose has been determined by (3). When it is estimated that $V_{t}$ will fall below the dead volume or is too large to waste, one can choose another dilution, that is, $V_{0}$.

To obtain values of parameters $\alpha$ and $\psi$ for a particular nebulization process a calibration experiment has to be performed by determining the initial values $V_{0}, C_{0}$ and measuring the final values $V_{t}, C_{t}$, at an appropriate time $t$. Then,

$\psi=\frac{V_{0}-V_{t}}{t} \times \frac{\ln \left(C_{t} / C_{0}\right)}{\ln \left(V_{0} / V_{t}\right)}$

$\alpha=\frac{V_{0}-V_{t}}{t}-\psi$ 
Table 1

Concentration $(\mathrm{mg} / \mathrm{ml})$ in nebulization chamber during nebulization of salbutamol, initial concentration $1.0 \mathrm{mg} / \mathrm{ml}$ with Heart ${ }^{\circledR}$ jet nebulizer, compressor flow of $101 / \mathrm{min}$

\begin{tabular}{clll}
\hline Time $(\mathrm{min})$ & Experimental & By $(1)^{\mathrm{b}}$ & \% Difference \\
\hline 0 & 1.00 & 1.00 & 0 \\
60 & 1.03 & 1.03 & 0 \\
120 & 1.05 & 1.07 & +2 \\
180 & 1.11 & 1.12 & +1 \\
$240^{\mathrm{a}}$ & 1.18 & Calibrator & Calibrator \\
& & point & point \\
300 & 1.29 & 1.27 & -2 \\
360 & 1.48 & 1.40 & -6 \\
420 & 1.69 & 1.65 & -2 \\
\hline
\end{tabular}

Experimental data of Raabe et al. (1998) (experimental), predicted by proposed model (1) and difference between experimental and predicted.

a Taken as calibrator time point, with $V_{t}(t=240 \mathrm{~min})=120 \mathrm{ml}$.

b $V_{0}=240 \mathrm{ml}, C_{0}=1 \mathrm{mg} / \mathrm{ml}$; by (5) and (6): $\psi=0.12 \mathrm{ml} / \mathrm{min}$, $\alpha=0.38 \mathrm{ml} / \mathrm{min}$.

\section{Experimental validation of the model}

The postulated model was validated using the published experimental data obtained with the Heart $^{\circledR}$ jet nebulizer, operated under a compressor flow of $101 / \mathrm{min}$, nebulizing $1.0 \mathrm{mg} / \mathrm{ml}$ salbutamol (Raabe et al., 1998), see Table 1. The results show good correspondence between the postulated model and the experimental data.

\section{Comparison with the model of Coates}

Coates et al. describe unvented nebulization as a combination of loss of volume in the nebulization chamber and an increase in concentration of the fluid. (Coates et al., 1998a). In our notation, the formula of these authors for cumulative drug output of unvented nebulizers reads ${ }^{2}$ :

$D_{t}=C_{0} V_{0}-\left(V_{0}-h t\right)\left(C_{0} \mathrm{e}^{b t}\right)$

\footnotetext{
${ }^{2}$ Their published formula contains also the factors $g$ and $a$ and reads

$D_{t}=C_{0} V_{0}-\left\{V_{0}-(g+h t)\right\}\left(C_{0} a \mathrm{e}^{b t}\right)$.
}

However, the results of Coates et al. show $g$ to deviate not significantly from zero and $a$ to deviate not significantly from 1 , allowing our simplification to (1). where $h$ is the slope of the loss of volume per unit of time and $b$ is the natural logarithmic slope of the increase in concentration per unit of time.

These authors reported experimental data obtained with two jet nebulizers, using moisturized and nonmoisturized air and operated with different compressors, nebulizing a dilution of tobramycin, they compared the experimental results with their model.

We compared our postulated model with the model of these authors and with their experimental values, see Table 2. It appears that both models are in good agreement with each other and with the experimental results over the time period until intermitted aerosol production.

\section{Dependency of efficiency on nebulization conditions}

Carrying out a calibration experiment for each new nebulization process is a tedious task. However, experimental data taken from literature show that the efficiency of an unvented jet nebulization process of diluted aqueous solutions is relatively insensitive to small changes in the concentration and to small changes in aspiration flow.

Data obtained by Le Brun et al. (1999b), with tobramycin show that an increase in concentration from 50 to $100 \mathrm{mg} / \mathrm{ml}$ does not influence the efficiency of the process, see Table 3. Note that in these experiments the Porta-Neb Ventstream ${ }^{\circledR}$ was used, a breath-enhanced jet nebulizer, but operated here in an unvented way. At higher concentrations of tobramycin larger changes in efficiency are found, caused by increasing viscosity, density and surface tension.

Also, a change of a factor two in the aspiration flow had little influence on the efficiency of the nebulization process of $100 \mathrm{mg} / \mathrm{ml}$, whereas larger changes are found at concentrations above $100 \mathrm{mg} / \mathrm{ml}$, see Table 3.

On the other hand, experimental literature data show that the efficiency of an unvented jet nebulization process is sensitive to the humidity of the compressor gas. The efficiencies of the nebulization processes described by Coates et al. (1998a) were calculated, see Table 4. It appears that the efficiency 
Table 3

Efficiencies of nebulization processes at different concentrations and aspiration flows data from Le Brun et al. (1999b)

\begin{tabular}{lll}
\hline $\begin{array}{l}\text { Concentration } \\
(\mathrm{mg} / \mathrm{ml})\end{array}$ & $\begin{array}{l}\text { Aspiration } \\
\text { flow 20 1/min }\end{array}$ & $\begin{array}{l}\text { Aspiration } \\
\text { flow 40 1/min }\end{array}$ \\
\hline 50 & 0.81 & \\
100 & 0.80 & 0.81 \\
200 & 0.95 & 0.85 \\
300 & 0.87 & 0.82 \\
\hline
\end{tabular}

Nebulized solution: tobramycin sulphate in water. Nebulizer: Porta-Neb Ventstream ${ }^{\circledR}$ operated unvented by continuous artificial aspiration. Compressor flow: 7.01/min (Le Brun, personal communication). Efficiencies calculated by postulated model.

Table 4

Efficiencies of nebulization processes described by Coates et al. (1998a)

\begin{tabular}{|c|c|}
\hline Nebulization process & Efficiency \\
\hline Hudson ${ }^{\circledR} 1720$, air, compressor flow $61 / \mathrm{min}$ & 0.65 \\
\hline Hudson ${ }^{\circledR} 1720$, air, compressor flow $81 / \mathrm{min}$ & 0.68 \\
\hline Hudson ${ }^{\circledR}$ 1720, moisturized air, Pulmo-Aide & 0.87 \\
\hline Hudson ${ }^{\circledR} 1730$, air, compressor flow $61 / \mathrm{min}$ & 0.85 \\
\hline Hudson ${ }^{\circledR} 1730$, air, compressor flow $81 / \mathrm{min}$ & 0.83 \\
\hline Hudson ${ }^{\circledR}$ 1730, moisturized air, Pulmo-Aide & 0.93 \\
\hline
\end{tabular}

See for explanation Table 2. Efficiencies calculated by postulated model.

is relatively insensitive to the compressor flow, but is sensitive to prior moisturization of the jet gas, as was also concluded by Coates et al. (1998a).

\section{Conclusion}

The presented physical model and derived formulas are useful tools for the quantification of nebulizer cumulative drug output over time of unvented jet nebulizers. The concept of efficiency is a useful parameter to compare nebulization processes. It should be noted that the model is validated for unvented jet nebulizers only. It might be expected that the model is also valid for unvented ultrasonic nebulizers as the physical principles underlying our model will also pertain to this class of nebulizers. However, the drug output of vented nebulizers is more complex due to the inspiratory flow (Coates et al., 1998b). 


\section{Appendix A. Analysis of the two compartment model}

We consider a simple two compartment model to analyze the nebulizer situation. The parameters have the meaning explained in the main body of the paper. For mathematical convenience we denote the time-dependent parameters as functions of time $t$ rather than parameters subscripted by time, that is, we write " $V(t)$ " for " $V_{t}$ " and " $C(t)$ " for " $C_{t}$." We assume that $\alpha$ and $\psi$ are constants independent of the current drug concentration and volume.

The model consists of two compartments: the nebulizer solution and the aerosol. The nebulizer solution has two parameters that are functions of time $t$, the volume $V(t)$ of the solution contained by the nebulizer, and its drug concentration $C(t)$. The interaction between the two compartments is given by a constant flow $\alpha$ of drug-containing solvent, and a constant flow $\psi$ of drugless solvent, from the nebulizer solution to the aerosol.

\section{A.1. Nebulizer volume over time (4)}

The volume of solution decreases during nebulization from the initial volume $V(0)$ at time 0 to $V(t)$ at time $t$. The difference $V(t)-V(0)$ must be equal to total flow of volume from the nebulizer solution to the aerosol from time 0 up to time $t$ :

$$
\begin{aligned}
V(t)-V(0) & =\int_{i=0}^{\mathrm{t}} \mathrm{d} V(i)=\int_{i=0}^{t}-(\alpha+\psi) \mathrm{d} i \\
& =-\left.(\alpha+\psi) i\right|_{i=0} ^{t}=-(\alpha+\psi) t
\end{aligned}
$$

Hence,

$V(t)=V(0)-(\alpha+\psi) t$.

Evident boundary conditions are that $V(t) \geq 0$ for all times $t$ considered-the volume of the solution cannot become negative. This restricts the range of (A.1) and the later formulas to times $t \leq V(0) /(\alpha+\psi)$. In fact, at the highest value allowed for $t$ the drug concentration $C(t)$ in (A.5) becomes infinite. This is as it should be: there is a positive amount of drug left at the time when the volume of the solution has vanished. Practically speaking, we can expect the formula to loose accuracy close to this extreme.

\section{A.2. Drug concentration over time (1)}

As usual in the calculus, let " $\Delta x$ " denote a small change in variable $x$ : from $x$ to $x+\Delta x$. Write

$C(x+\Delta x)=C(x)+\Delta C(x)$,

$V(x+\Delta x)=V(x)+\Delta V(x)$,

where $\Delta C(x)$ is the change in concentration from time $x$ to time $x+\Delta x$, and $\Delta V(x)$ is the change in volume of nebulizer solution between those times. The quantity of drug in the nebulizer at time $x$ is $C(x) V(x)$ and at time $x+\Delta x$ it is $C(x+\Delta x) V(x+\Delta x)$. The difference between the two quantities has been transported from the nebulizer solution to the aerosol in the time interval $\Delta x$. The flow of drug-containing solvent to the aerosol is constant at $\alpha$. Therefore, the total change in drug quantity in the time interval $\Delta x$ is

$C(x+\Delta x) V(x+\Delta x)-C(x) V(x)=-\alpha C(x) \Delta x$,

and the change in volume of nebulizer solution is $\Delta V(x)=-(\alpha+\psi) \Delta x$. Hence,

$V(x+\Delta x)=V(x)+\Delta V(x)=V(x)-(\alpha+\psi) \Delta x$,

where we assume that $\Delta x$ is small enough to validate the assumption that $C\left(x^{\prime}\right)$ and $V\left(x^{\prime}\right)$ can be taken as constant for $x^{\prime} \in(x, x+\Delta x)$. Then, we can rewrite $C(x+\Delta x) V(x+\Delta x)$ in two ways: firstly according to (A.2), and secondly by substituting the $V(x+\Delta x)$ factor from (A.3), to obtain

$$
\begin{aligned}
C(x) V(x)-\alpha C(x) \Delta x= & C(x+\Delta x)(V(x) \\
& -(\alpha+\psi) \Delta x) .
\end{aligned}
$$

Let $\Delta x \rightarrow 0$, which is the same as replacing it by the infinitesimal interval $d x$. Rearranging the resulting equation

$C(x+\mathrm{d} x)=\frac{C(x) V(x)-\alpha C(x) \mathrm{d} x}{V(x)-(\alpha+\psi) \mathrm{d} x}$.

Write $\mathrm{d} C(x)=C(x+\mathrm{d} x)-C(x)$ and substitute the right-hand side of the above equation for $C(x+\mathrm{d} x)$ to obtain:

$$
\begin{aligned}
\mathrm{d} C(x) & =\frac{C(x) V(x)-\alpha C(x) \mathrm{d} x}{V(x)-(\alpha+\psi) \mathrm{d} x}-C(x) \\
& =\frac{\psi C(x) \mathrm{d} x}{V(\mathrm{x})-(\alpha+\psi) \mathrm{d} x}
\end{aligned}
$$


Observing that $V(x)-(\alpha+\psi) \mathrm{d} x \rightarrow V(x)$ for $\mathrm{d} x \rightarrow$ 0 yields:

$\mathrm{d} C(x)=\frac{\psi C(x)}{V(x)} \mathrm{d} x$.

Rearranging and integrating,

$\int_{x=0}^{t} \frac{1}{C(x)} \mathrm{d} C(x)=\int_{x=0}^{t} \frac{\psi}{V(x)} \mathrm{d} x$.

Integrating the left-hand side gives

$\int_{x=0}^{t} \frac{1}{C(x)} \mathrm{d} C(x)=\left.\ln C(x)\right|_{x=0} ^{t}=\ln \frac{C(t)}{C(0)}$

The argument in the derivation of (A.1) also supports $\mathrm{d} V(x)=-(\alpha+\psi) \mathrm{d} x$. Integrating the right-hand side gives (use $\mathrm{d} V(x) / \mathrm{d} x=-(\alpha+\psi)$ in the second step, and (A.1) in the fourth step)

$$
\begin{aligned}
& \int_{x=0}^{t} \frac{\psi}{V(x)} \mathrm{d} x=\int_{V(x)=V(0)}^{V(t)} \frac{\psi}{V(x)} \frac{1}{\mathrm{~d} V(x) / \mathrm{d} x} \mathrm{~d} V(x) \\
& \quad=-\frac{\psi}{\alpha+\psi} \int_{V(x)=V(0)}^{V(t)} \frac{1}{V(x)} \mathrm{d} V(x) \\
& =-\left.\frac{\psi}{\alpha+\psi} \ln V(x)\right|_{V(x)=V(0)} ^{V(t)} \\
& =-\frac{\psi}{\alpha+\psi}(\ln (V(0)-(\alpha+\psi) t)-\ln V(0)) \\
& =-\frac{\psi}{\alpha+\psi} \ln \left(\frac{V(0)-(\alpha+\psi) t}{V(0)}\right)
\end{aligned}
$$

Since the right-hand side is equal to the left-hand side in (A.4), we finally obtain

$C(t)=C(0)\left(\frac{V(0)}{V(0)-(\alpha+\psi) t}\right)^{\psi /(\alpha+\psi)}$,

that is, (1).

\section{A.3. Accumulative nebulized dose over time (2)}

The parameters $\alpha$ and $\psi$ are assumed to be constant. This assumption is only an approximation as is further discussed in the main text.

Integrating over the nebulization time gives the expression for the total drug dose that is nebulized. Without loss of generality, we assume $\alpha \neq 0$. This is a realistic restriction, since this is required to deliver any quantity of drug at all.
The total dose $D(t)$ delivered by continuous nebulization from time 0 to time $t$ is given by (substitute $C(x)$ from (A.5)):

$$
\begin{aligned}
D(t) & =\int_{x=0}^{t} \alpha C(x) \mathrm{d} x \\
& =\alpha C(0) \int_{x=0}^{t}\left(\frac{1}{1-((\alpha+\psi) / V(0)) x}\right)^{\psi /(\alpha+\psi)} \mathrm{d} x .
\end{aligned}
$$

Change to a new variable $y=1-((\alpha+\psi) / V(0)) x$. Then, $\mathrm{d}(y)=-((\alpha+\psi) / V(0)) \mathrm{d} x$, and the lower integration bound 0 becomes 1 , and upper integration bound $t$ becomes $1-((\alpha+\psi) / V(0)) t$, respectively. Therefore, rewriting $(1-(\psi /(\alpha+\psi)))=\alpha /(\alpha+\psi)$ in the second step, both in the resulting leading coefficient and in the exponent, we found:

$$
\begin{aligned}
D(t) & =-\frac{\alpha C(0) V(0)}{\alpha+\psi} \int_{y=1}^{1-((\alpha+\psi) / V(0)) t} y^{-\psi /(\alpha+\psi)} \mathrm{d} y \\
& =-\left.\left(\frac{\alpha+\psi}{\alpha}\right) \frac{\alpha C(0) V(0)}{\alpha+\psi} y^{\alpha /(\alpha+\psi)}\right|_{y=1} ^{1-((\alpha+\psi) / V(0)) t} \\
& =C(0) V(0)\left[1-\left(\frac{V(0)-(\alpha+\psi) t}{V(0)}\right)^{\alpha /(\alpha+\psi)}\right]
\end{aligned}
$$

which asserts (2).

\section{A.4. Time to administer a given dose (3) and remainder volume}

Let $D$ be the total dose in milligram of the drug nebulized at time $t_{D}$ starting from time 0 . Moreover, let $V_{D}$ be the remainder volume at the time $t_{D}$. When $D$, $C(0), V(0), \alpha$, and $\psi$ are known, we can solve $t_{D}$ and $V_{D}$ simply analytically. In (2), we set $D=D(t)$ and $t=t_{D}$. Solving the resulting equation for $t_{D}$, we find:

$t_{D}=\frac{V(0)}{\alpha+\psi}\left[1-\left(1-\frac{D}{C(0) V(0)}\right)^{1+\psi / \alpha}\right]$.

The remainder solution volume $V_{D}=V\left(t_{D}\right)$ left after administering a total dose $D$ is now given by (A.1), substituting the right-hand side of (A.6) for $t_{D}$ :

$$
\begin{aligned}
V_{D} & =V(0)-(\alpha+\psi) t_{D} \\
& =V(0)\left(1-\frac{D}{C(0) V(0)}\right)^{1+\psi / \alpha} .
\end{aligned}
$$




\section{A.5. Efficiency 1 gives linearity}

The ratio $\alpha /(\alpha+\psi)$ is called the efficiency of the nebulizer. An efficiency of 1 means that $\psi=0$, that is, there is no loss due to vaporized solvent not containing drug. Corresponding substitution $\psi=0$ in (2) and (3) gives

$D=C(0) \alpha t_{D}$

$t_{D}=\frac{D}{\alpha C(0)}$.

That is, the nebulizing process becomes linear: the concentration in the nebulizing solution does not change over time, and the delivered drug dose is linear in the delivery time, and vice versa.

\section{A.6. Expressions (6) and (5) for $\alpha$ and $\psi$}

Rearranging (A.1) yields

$\alpha+\psi=\frac{V(0)-V(t)}{t}$,

which implies (6).

The relation between $\psi$ and the concentration and volume over time is given by (A.5). Rearranging the formula by bringing $C(0)$ in the denominator of the left-hand side and taking the logarithm on both sides of the resulting equation yields

$\ln \frac{C(t)}{C(0)}=\frac{\psi}{\alpha+\psi} \ln \frac{V(0)}{V(0)-(\alpha+\psi) t}$.
Substituting $\alpha+\psi$ according to (A.7) and rearranging, we find

$\psi=\frac{V(0)-V(t)}{t} \frac{\ln C(t) / C(0)}{\ln V(0) / V(t)}$,

which proves (5).

\section{References}

Coates, A.L., Macneish, C.F., Lands, L.C., Smountas, A., Meisner, D., Kelemen, S., Vadas, E.B., 1998a. Factors influencing the rate of drug output during the course of wet nebulization. J. Aerosol. Med. 11, 101-111.

Coates, A.L., MacNeish, C.F., Lands, L.C., Meisner, D., Kelemen, S., Vadas, E.B., 1998b. A comparison of the availability of tobramycin for inhalation from vented vs. unvented nebulizers. Chest 113, 951-956.

Le Brun, P.P.H., De Boer, A.H., Gjaltema, D., Hagedoorn, P., Heijerman, H.G.M., Frijlink, H.W., 1999a. Inhalation of tobramycin in cystic fibrosis. Part 1: the choice of a nebulizer. Int. J. Pharm. 189, 205-214.

Le Brun, P.P.H., De Boer, A.H., Gjaltema, D., Hagedoorn, P., Heijerman, H.G.M., Frijlink, H.W., 1999b. Inhalation of tobramycin in cystic fibrosis. Part 2: optimization of the tobramycin solution for a jet and an ultrasonic nebulizer. Int. J. Pharm. 189, 215-225.

Raabe, O.G., Wong, T.W., Wong, G.B., Roxburgh, J.W., Piper, S.D., Lee, J.I.C., 1998. Continuous nebulization therapy for asthma with aerosols of $\beta_{2}$ agonists. Ann. Allergy Asthma Immunol. 80, 499-508.

\section{Further reading}

Le Brun, P.P.H., De Boer, A.H., Heijerman, H.G.M., Frijlink, H.W., 2000. A review of the technical aspects of drug nebulization. Pharm. World Sci. 22, 75-81. 